\title{
NUTRITION Acides gras polyinsaturés de microalgues : intérêts et développements actuels
}

Nutrition Benefits and current developments of polyunsaturated fatty acids from microalgae lipids

Oléagineux, Corps Gras, Lipides. Volume 9, Numéro 2, 92-5, Mars - Juin 2002, La filière

Auteur(s) : Laurent POISSON, Marie DEVOS, Gaëlle PENCREAC'H, Françoise ERGAN, Laboratoire d'application des lipases de I'université du Maine, Institut universitaire de technologie de Laval, Département génie biologique, 52, rue Calmette-et-Guérin, BP 2045, 53020 Laval Cedex 9, France.

Author(s) : Laurent POISSON, Marie DEVOS, Gaëlle PENCREAC'H, Françoise ERGAN

Résumé : Commercialisés comme compléments nutritionnels sous forme de capsules ou utilisés comme ingrédients pour la préparation de lait maternisé, les acides gras polyinsaturés (AGPI) de la série ôméga3 sont principalement obtenus à partir d'huiles de poisson. Du fait de leur richesse en AGPI, les microalgues constituent une source alternative à fort potentiel pour l'obtention de ces acides gras d'intérêt. Différents travaux, cherchant à améliorer la productivité des photobioréacteurs et les procédés de purification, ont largement contribué à réduire les coûts de production d'AGPI d'origine microalgale. Toutefois, pour rendre les microalgues compétitives par rapport aux huiles de poisson pour l'obtention d'AGPI, des travaux d'exploration métabolique, de biologie moléculaire et de génie enzymatique sont encore à mener.

Summary : Commercialized as nutraceuticals under the form of capsules or used as ingredients in infant food products, ôméga3 polyunsaturated fatty acids (PUFAs) are mainly obtained from fish oil. Due to their high contain in PUFAs, microalgae are considered as a good alternative source of these fatty acids of interest. Several studies leading to an improvement of photobioreactors and purification processes have widely contributed to reduce the costs of PUFA production from microalgae lipids. Nevertheless, in order to be economically attractive, PUFAs from microalgae need a convergence of genetic, metabolic and enzymatic trials.

Mots-clés : AGPI, microalgues.

Keywords : PUFAs, microalgae.

\section{ARTICLE}

L'intérêt des acides gras polyinsaturés

Grâce aux travaux épidémiologiques de Dyerberg et Bang [1], il a été montré que les acides gras polyinsaturés (AGPI) de la série ôméga3 apportent une protection contre les maladies cardiovasculaires. Les AGPI sont également des éléments clés dans le développement du cerveau et 
de la rétine [2] et, à ce titre, un lait maternisé supplémenté en acide docosahexaénoïque (DHA, 22:6 ôméga3) est recommandé pour les prématurés [3]. Commercialisés comme compléments nutritionnels, les AGPI possèdent un intérêt économique important : I'acide eicosapentaénoïque (EPA, 20:5 ôméga3) et le DHA, principalement conditionnés en capsules, sous forme de triglycérides et d'esters éthyliques, représenteraient un marché annuel mondial supérieur à 915 millions d'euros chacun [4], soit un prix au kilo de produit (pur à $95 \%$ ) de 650 dollars [5].

\section{Les sources d'AGPI}

Les AGPI à chaînes longues comme I'EPA et le DHA peuvent être fournis à l'organisme par l'alimentation ou synthétisés de novo à partir des deux acides gras essentiels que sont les acides linoléique (18:2 ôméga6) et alpha-linolénique (18:3 ôméga3).

La principale source d'AGPI à chaîne longue comme le DHA ou l'EPA est, jusqu'à présent l'huile de poisson (morue, hareng, sardine, saumon, thon) [6], un co-produit bon marché de l'industrie de la pêche. Cette source est par ailleurs disponible en quantité importante puisque la production mondiale annuelle d'huile de poisson est évaluée à 1 million de tonnes [7].

Toutefois, dans une publication [7] fréquemment citée dans la littérature, Yongmanitchai et Ward ont fait part, en 1989, de l'intérêt des microalgues comme source potentielle d'AGPI. En effet, à la base de la chaîne alimentaire marine, les microalgues peuvent posséder des teneurs en AGPI pouvant atteindre jusqu'à $50 \%$ des acides gras totaux [8-12] (tableau) et $6 \%(\mathrm{~m} / \mathrm{m})$ de la biomasse sèche.

\section{L'intérêt des microalgues pour la production d'AGPI}

Contrairement aux huiles de poisson, les lipides de microalgues ne contiennent pas de cholestérol [6], d'où une meilleure adaptation pour des applications thérapeutiques visant à réduire le taux de lipides sériques. De par leur statut de cellule végétale, les microalgues possèdent une image favorable auprès des consommateurs, ce qui facilite leur utilisation comme ingrédients de produits cosmétiques ou alimentaires. Cela est par ailleurs renforcé par l'absence de l'odeur désagréable dégagée par les huiles de poisson [6].

De plus, la culture des microalgues en bioréacteur permet de s'affranchir du problème de l'approvisionnement que peuvent connaître les produits issus de la pêche. On peut d'ailleurs souligner que, contrairement à la culture d'autres micro-organismes producteurs d'AGPI comme les champignons du genre Mortierella, la culture de microalgues ne nécessite que des substrats peu coûteux comme de l'eau de mer, du $\mathrm{CO}_{2}$, de l'énergie solaire et quelques minéraux (figure 1).

Constitués non seulement de triglycérides mais également de phospholipides et de glycolipides, les lipides de microalgues offrent un large éventail d'applications. Des fractions de phospholipides riches en AGPI peuvent par exemple s'avérer plus intéressantes pour certaines applications médicales que des fractions de triglycérides riches en AGPI puisque c'est associés aux phospholipides membranaires qu'on retrouve les AGPI au niveau de la rétine et du cerveau. Du fait de leurs propriétés 
émulsifiantes, les phospholipides sont, de plus, souvent préférés aux autres classes de lipides pour la formulation des ingrédients au niveau industriel.

La pertinence de l'utilisation des microalgues comme source d'AGPI réside également dans la possibilité d'exploiter de manière concomitante d'autres produits à haute valeur ajoutée comme l'astaxanthine, le beta-carotène et la phycoérythrine, utilisés comme colorants alimentaires ou la superoxyde dismutase, un antioxydant d'intérêt pharmaceutique ou encore le sulfoquinovosyldiacylglycérol (SQDG), un constituant des glycolipides présentant une activité antitumorale (photo) $[13,14]$.

Les limites techniques et économiques

Les difficultés techniques et économiques restent encore à ce jour les limites majeures à l'utilisation des microalgues comme sources d'AGPI. Le coût de production d'AGPI à partir de lipides de microalgues est en effet encore prohibitif par rapport à l'obtention d'AGPI à partir d'huile de poisson et ce aussi bien au niveau du coût de la matière première que du coût de la purification [5].

En ce qui concerne la matière première, si le milieu de culture est peu coûteux, l'éclairement nécessaire des cellules est une source de difficultés au niveau de la conception des bioréacteurs. Différentes stratégies ont été développées pour la production de biomasse microalgale [4, 15-21]. La première de ces stratégies est la culture extensive en extérieur dans des bassins ouverts de forme circulaire ou en boucle (raceway) construits avec des matériaux peu coûteux comme des bâches plastiques. Cette méthode de production est cependant peu productive et ne permet pas l'obtention de cultures axéniques. Elle est, de plus, presque uniquement accessible aux régions bénéficiant d'un ensoleillement important sur une majeure partie de l'année comme les États-Unis, l'Australie, la Thaïlande ou Israël. Les lipides de microalgues étant d'autre part sujets à des variations qualitatives et quantitatives en fonction du milieu de culture, du temps de séjour des cellules et des conditions environnementales, des problèmes de maîtrise de la production sont rencontrés avec ce type de réacteur soumis aux aléas météorologiques. Des photobioréacteurs fermés, tubulaires ou plans ont été développés au niveau pilote, notamment par Gudin et Chaumont au CEA de Cadarache [20]. Ces systèmes, constitués de tubes ou de lamelles de plexiglas dans lesquels circulent les microalgues, ont considérablement amélioré la maîtrise des conditions de culture et la productivité du fait d'un meilleur éclairement des cellules. Ils sont toutefois plus gourmands en énergie (donc plus coûteux) et difficilement transposables à l'échelle industrielle du fait de limites techniques comme le transfert en $\mathrm{CO}_{2} / \mathrm{O}_{2}$ à l'intérieur des tubulures ou des lamelles de plexiglas.

Afin de pallier les difficultés de conception associées aux photobioréacteurs, l'utilisation de microalgues hétérotrophiques comme Crypthecodinium cohnii [12], cultivables sans éclairement, a été préconisée. Toutefois, si cette alternative constitue une manière originale de valoriser les microalgues marines, elle rend caduque l'utilisation de substrats peu coûteux qui, comme cela a été précisé précédemment, est l'un des intérêts des microalgues pour la production d'AGPI.

Au niveau de la purification (figure 2), qui représente généralement près de $70 \%$ du coût de production, des difficultés supplémentaires sont rencontrées avec les lipides de microalgues par rapport aux huiles de poisson. Une dépigmentation des extraits lipidiques microalgaux est en effet nécessaire [5] et occasionne une étape supplémentaire de séparation par passage sur charbon actif, gel de silice ou encore par extraction au fluide supercritique. Les pigments, comme la chlorophylle, 
sont extraits de la biomasse microalgale en même temps que les lipides et constituent une contrainte importante pour l'exploitation des AGPI de microalgues, en particulier pour leur utilisation comme ingrédients de produits alimentaires.

De plus, à partir des poissons, il est possible d'obtenir une huile relativement homogène, presque exclusivement composée de triglycérides ou de phospholipides, en sélectionnant des tissus et organes particuliers (foie, tête, peau, tissu adipeux, etc.). Cela est bien sûr impossible avec les microalgues et conduit également à augmenter le nombre d'étapes de purification et à faire parfois appel à des techniques élaborées [22] afin de séparer glycolipides, phospholipides, triglycérides, voire certaines autres sous-espèces moléculaires comme la phosphatidylcholine ou le SQDG.

L'avenir

En 2000, après 3 ans de recherche, un photobioréacteur industriel a été mis en place par la société Preussag à Wolfsburg en Allemagne. Ce réacteur de $700 \mathrm{~m}^{3}$, constitué de $500 \mathrm{~km}$ de tubulures de plexiglas, permettrait d'atteindre une production annuelle de 130 à 150 tonnes de biomasse sèche [18]. Ce type de développement industriel particulièrement encourageant devrait à terme rendre la biomasse microalgale meilleur marché et plus attractive par rapport aux huiles de poisson.

Toutefois, pour être pleinement valorisées, les améliorations réalisées au niveau de la production de biomasse doivent être associées à une convergence d'études à la fois biochimiques et génétiques. Le criblage de mutants métaboliques devrait par exemple permettre d'obtenir des souches hautement productrices ou présentant des profils en acides gras originaux par rapport à la souche sauvage. $\mathrm{Ce}$ dernier point est illustré dans le tableau, avec la souche CCAP 927/1 et le mutant métabolique ALII4 d'Isochrysis galbana. Comme cela a déjà été entrepris avec Porphyridium cruentum [10] et Haslea ostrearia [23], l'exploration de la biosynthèse des AGPI chez les microalgues semble être également une autre voie importante de développement, en particulier si ces études sont relayées par des travaux de biologie moléculaire permettant, à terme, d'agir directement au niveau du génome sur la production d'acides gras. Enfin, un traitement enzymatique des lipides de microalgues (figure 3) visant l'obtention de fractions enrichies en AGPI devrait permettre de réduire le nombre d'étapes de purification ainsi que la consommation en énergie [8, 24].

\section{REFERENCES}

1. KINSELLA JE (1986). Dietary fish oils. Possible effects of n-3 polyunsaturated fatty acids in reduction of thrombosis and heart disease. Nutrition Today, November/December : 7-14.

2. SANGIOVANNI JP, BERKEY CS, DWYER JT, COLDITZ GA (2000). Dietary essential fatty acids, longchain polyunsaturated fatty acids, and visual resolution acuity in healthy fullterm infants: a systematic review. Early Hum Dev, 57 : 165-88.

3. CRASTES DE PAULET A (1994). Importance des apports lipidiques pour le développement psychomoteur du petit enfant : insuffisance en acides gras polyinsaturés des laits premier âge. $O C L, 1$ : 15-7.

4. CORNET JF (1998). Le technoscope : les photobioréacteurs. Biofutur, $176: 1-10$. 
5. BELARBI EH, MOLINA GRIMA E, CHISTI Y (2000). A process for high yield and scaleable recovery of high purity eicosapentaenoic acid esters from microalgae and fish oil. Proc Biochem, 35 : 951-69.

6. ROBLES MEDINA A, MOLINA GRIMA E, GIMENEZ GIMENEZ A, IBANEZ GONZALEZ MJ (1998). Downstream processing of algal polyunsaturated fatty acids. Biotechnol Adv, 16 : 517-80.

7. YONGMANITCHAI W, WARD OP (1989). Omega-3 fatty acids: alternative sources of production. Proc Biochem, 24 : 117-25.

8. POISSON L, ERGAN F (2001). Docosahexaenoic acid ethyl esters from Isochrysis galbana. J Biotechnol, $91: 75-81$.

9. MOLINA GRIMA E, SANCHEZ PÉREZ JA, GARCIA CAMACHO F, ROBLES MEDINA A, GIMÉNEZ GIMÉNEZ A, LOPEZ ALONSO D (1995). The production of polyunsaturated fatty acids by microalgae: from strain selection to product purification. Proc Biochem, 30 : 711-9.

10. SHIRAN D, KHOZIN I, HEIMER YM, COHEN Z (1996). Biosynthesis of eicosapentaenoic acid in the red microalga Porphyridium cruentum. I: The use of externally supplied fatty acids. Lipids, 31 : 127782.

11. BERGÉ JP, GOUYGOU JP, DUBACQ JP, DURAND P (1995). Reassessment of lipid composition of the diatom, Skeletonema costatum. Phytochemistry, $39: 1017-21$.

12. JIANG Y, CHEN F, LIANG S-Z (1999). Production potential of docosahexaenoic acid by the heterotrophic marine dinoflagellate Crypthecodinium cohnii. Proc Biochem, 34 : 633-7.

13. VILCHEZ C, GARBAYO I, LOBATA MV, VEGA JM (1997). Microalgae-mediated chemicals production and wastes removal. Enzyme Microb Technol, 20 : 562-72.

14. QUASNEY ME, CARTER LC, OXFORD C, WATKINS SM, GERSHWIN ME, GERMAN JB (2001). Inhibition of proliferation and induction of apoptosis in SNU-1 human gastric cancer cells by the plant sulfolipid, sulfoquinovosyldiacylglycerol. J Nutr Biochem, 12 : 310-5.

15. BECKER EW (1994). Microalgae: biotechnology and microbiology. Cambridge : Cambridge University Press ; 295 p.

16. BOROWITZKA MA (1999). Commercial production of microalgae: ponds, tanks, tubes and fermenters. J Biotechnol, 70 : 313-21.

17. MÜLLER-FEUGA A, BARON R, LE GUÉDES R, MICHEL E (1998). Theoretical assessment of productivity in photobioreactors by radiative transfer approach. In : Le Gal Y, Müller-Feuga A, eds. Marine microorganisms for industry. Plouzané : Ifremer, 194-201.

18. PULZ O (2001). Photobioreactors: production systems for phototrophic microorganisms. Appl Microbiol Biotechnol, 57 : 287-93.

19. PULZ O, SCHEIBENBOGEN K, GROB W (2001). Biotechnology with cyanobacteria and microalgae. In : Rehm HJ, Reed G, eds. Biotechnology. Weinheim : WILEY-VCH, 105-36.

20. VONSHAK A (1988). Porphyridium. In : Borowitzka MA, Borowitzka LJ, eds. Micro-algal 
Biotechnology. Cambridge : Cambridge University Press, 122-34.

21. TREDECI MR, ZITTELLI GC (1998). Efficiency of sunlight utilization: tubular versus flat photobioreactors. Biotechnol Bioeng, 57 : 187-97.

22. BAUDIMANT G, GOUYGOU JP, DURAND P (2000). Purification et caractérisation de la phosphatidylcholine de la microalgue Porphyridium cruentum par couplage chromatographique de partage centrifuge (CPC) et chromatographie couche mince avec formateur de gradient (HPTLCAMD). In : Baudimant G, Guézennec J, Roy P, Samain F, eds. Marine lipids. Plouzané : Ifremer, 30-1.

23. ULMANN L, MIMOUNI V, TREMBLIN G (2002). Biosynthèse des acides gras à longue chaîne chez Haslea ostrearia Simonsen. Influence du niveau d'éclairement. Crypt Algol (sous presse).

24. POISSON L, JAN S, ERGAN F (2000). Study on lipase-catalysed esterification of arachidonic acid in view of further PUFA enrichment of microalgae lipid extracts. In : Baudimant G, Guézennec J, Roy P, Samain JF, eds. Marine lipids. Plouzané : Ifremer, 204-11.

Illustrations

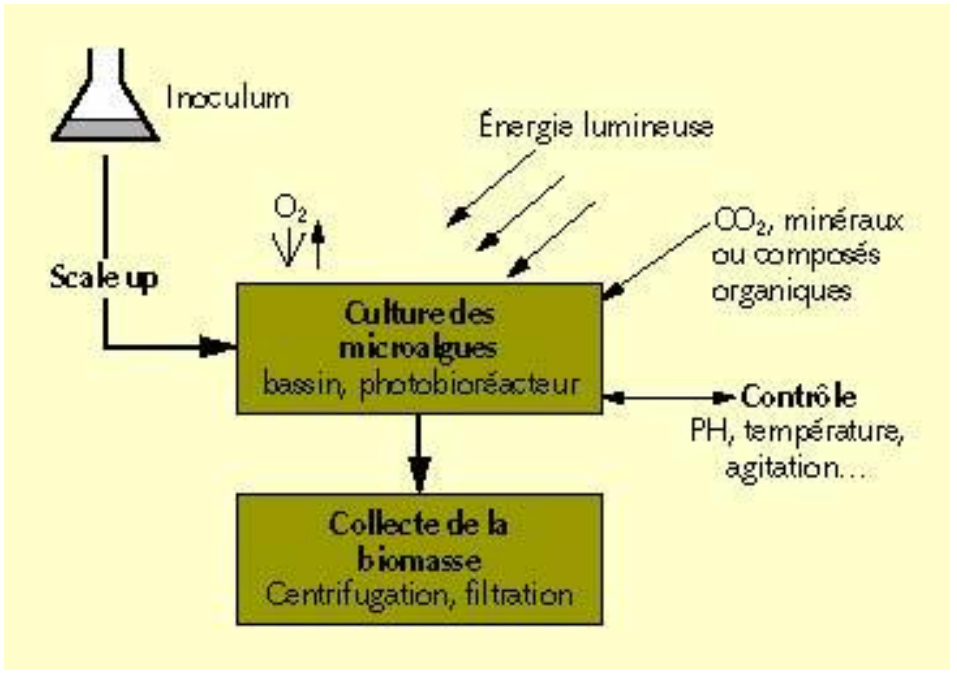

Figure 1. Production de microalgues (d'après [19]). 




Figure 2. Exemple de procédé de purification d'AGPI de microalgues (d'après [5]). 


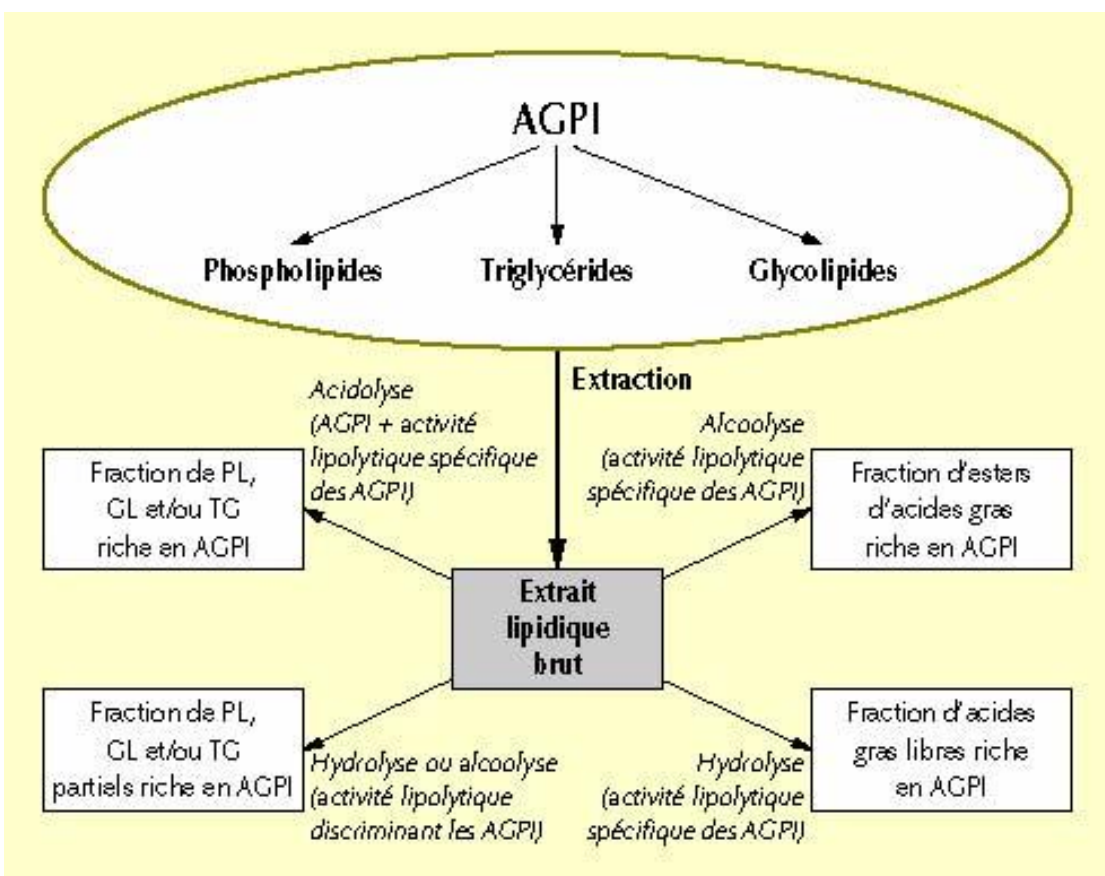

Figure 3. Stratégies de traitement enzymatique des lipides de microalgues pour I'obtention de fractions enrichies en AGPI.

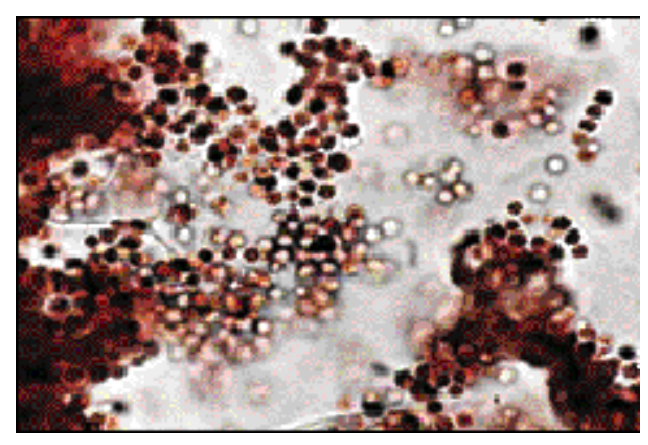

Photo. Cénobe de Porphyridium cruentum. Productrice d'acides arachidonique (20:4 ôméga3) et eicosapentanoïque (20:5 ôméga3), cette microalgue est également intéressante pour ses polysaccharides et ses pigments. 
Tableau. Profil en acides gas de quelques mir oalgues.

\begin{tabular}{|c|c|c|c|c|c|}
\hline $\begin{array}{l}\text { Acid es gras } \\
(\%, m / m)\end{array}$ & $\begin{array}{c}\text { Isochrysis } \\
\text { galbana } \\
\text { CCAP 927/1 } \\
\text { [8] }\end{array}$ & $\begin{array}{c}\text { Isochrysis } \\
\text { galbana } \\
\text { ALll4 } \\
\text { [9] }\end{array}$ & $\begin{array}{l}\text { Porphyidium } \\
\text { cruentum } \\
\text { 1380.1d } \\
\text { [10] }\end{array}$ & $\begin{array}{c}\text { Skeletonema } \\
\text { costatum } \\
{[11]}\end{array}$ & $\begin{array}{l}\text { Crypthecodinium } \\
\text { cohnii } \\
\text { ATCC } 30556 \\
\text { [12] }\end{array}$ \\
\hline $12: 0$ & - & & - & - & 1,6 \\
\hline $14: 0$ & 13,5 & 10,7 & - & 5,4 & 13,0 \\
\hline $16: 0$ & 12,5 & 18,9 & 30,3 & 4,2 & 19,8 \\
\hline $16: 1$ & 1,4 & 23,2 & 6,4 & 11,2 & Trace \\
\hline $16: 2$ & - & - & - & 4,5 & - \\
\hline $16: 3$ & - & - & - & 13,1 & - \\
\hline $16: 4$ & - & - & - & 12,6 & - \\
\hline $18: 0$ & 1,2 & 0,5 & 0,5 & 0,3 & 12,8 \\
\hline $18: 1$ & 19,7 & 4,9 & 0,5 & 1,3 & 0,8 \\
\hline $18: 2$ & 6,0 & 0,9 & 5,3 & 1,5 & - \\
\hline $18: 3$ & 4,6 & 1,5 & 0,7 & - & - \\
\hline $18: 4$ & 7,7 & 7,3 & - & 4,4 & - \\
\hline $20: 4$ & - & 0,7 & 16,8 & - & - \\
\hline $20: 5$ & - & 22,6 & 37,9 & 35,3 & 51,1 \\
\hline $22: 6$ & 14,6 & 6,8 & - & 5,6 & \\
\hline Total ACPI & 32,9 & 39,8 & 60,7 & 51,3 & 51,1 \\
\hline
\end{tabular}

